\title{
THE USE OF AGGREGATE DATA TO ESTIMATE GOMPERTZ-TYPE OLD-AGE MORTALITY IN HETEROGENEOUS POPULATIONS
}

\author{
Christopher R. HeathCote ${ }^{1}$, Borek D. PuZA ${ }^{2 *}$ AND SteVen P. Roberts ${ }^{2}$ \\ Australian National University
}

\begin{abstract}
Summary
We consider two related aspects of the study of old-age mortality. One is the estimation of a parameterized hazard function from grouped data, and the other is its possible deceleration at extreme old age owing to heterogeneity described by a mixture of distinct sub-populations. The first is treated by half of a logistic transform, which is known to be free of discretization bias at older ages, and also preserves the increasing slope of the log hazard in the Gompertz case. It is assumed that data are available in the form published by official statistical agencies, that is, as aggregated frequencies in discrete time. Local polynomial modelling and weighted least squares are applied to cause-of-death mortality counts. The second, related, problem is to discover what conditions are necessary for population mortality to exhibit deceleration for a mixture of Gompertz sub-populations. The general problem remains open but, in the case of three groups, we demonstrate that heterogeneity may be such that it is possible for a population to show decelerating mortality and then return to a Gompertz-like increase at a later age. This implies that there are situations, depending on the extent of heterogeneity, in which there is at least one age interval in which the hazard function decreases before increasing again.
\end{abstract}

Key words: deceleration; Gompertz; heterogeneous populations; mortality; old age.

\section{Introduction}

The Gompertz model of ageing implies that the logarithm of the hazard function increases linearly with age. However, recent literature suggests that, at least for certain species, there may be a deceleration of the hazard function at older ages, in the sense of a decrease in its rate of increase or even a decrease in the function itself. That is to say, the Gompertz model does not necessarily hold at older ages for humans past the age of about 85. A feature of this phenomenon is that deceleration can occur in populations comprising individuals each of whom satisfies a Gompertz model. One may then ask how much heterogeneity is sufficient for deceleration to occur, a question taken up (but not resolved) towards the end of this paper.

The deceleration of mortality and heterogeneity have an extensive literature dating from Vaupel, Manton \& Stallard (1979). For recent discussion and a bibliography see, for example, Steinsaltz \& Wachter (2006), Gavrilov \& Gavrilova (2001), Horiuchi \& Wilmoth (1998), Wang, Müller \& Capra (1998) and Müller, Wang \& Capra (1997). The first two of these references pay particular attention to the approach based on frailty (described in Section 2), and especially to the Gamma-Gompertz proportional hazards model in which a Gamma

\footnotetext{
* Author to whom correspondence should be addressed.

${ }^{1}$ Centre for Mathematics and its Application, Australian National University, Canberra ACT 0200, Australia.

${ }^{2}$ School of Finance and Applied Statistics, Australian National University, Canberra ACT 0200, Australia. e-mail: borek.puza@anu.edu.au

Acknowledgements. We are indebted to Krys Sadkowsky of AIHW for providing the data used in Sections 4 and 5, and to Timothy Higgins for helpful discussions.
} 
distribution of frailties is used to define the mixing of Gompertz hazards. Carey \& Tuljapurkar (2003) discuss the importance of the question from the point of view of population biology. Gavrilov \& Gavrilova (2001) show how deceleration can be addressed by arguments from systems reliability theory, with the levelling of mortality at extreme old age explained as a process of simplification. Wang et al. (1998) and Müller et al. (1997) are mainly concerned with estimating a Gompertz-type hazard function at old ages when the age interval becomes small. In contrast, our concern will be with discrete age aggregate data with a fixed age interval, and we use population cause of death frequencies recently published by the Australian Institute of Health and Welfare.

Our description of heterogeneity is by mixture model, which can be deduced from the frailty formulation of Vaupel et al. (1979) without, however, relying on proportional hazards. Equation (5) below states the model as an expression for the population survivor function as a linear combination of sub-population survivor functions.

Although cases of deceleration are extensively reported, the possibility of resumption of acceleration after an episode of deceleration does not seem to have been raised. However, as shown in Section 5 below, there exist models of mixtures of Gompertz groups such that, depending on the extent of heterogeneity, there may be none, one or several age intervals of deceleration of the population hazard function interspersed with intervals of acceleration. Gompertz-like behaviour may then be resumed at extreme old age. An intuitive explanation is that deceleration occurs when the weakest group is dying out, followed by a brief reassertion of Gompertz acceleration before the next weakest group dies out, and so forth. Clearly it should be noted that the resumption of acceleration, if any, may occur at such an old age as to be impossible to check from data and hence be of only theoretical interest. On the other hand, for a human population, the example of Section 5 shows that cases could exist in which such episodes commence at about age 80. These remarks are not inconsistent with the third category listed on page 20 of Steinsaltz \& Wachter (2006).

The papers by Wang et al. (1998) and Müller et al. (1997) provide the point of departure for Sections 3 and 4. Their preferred statistic is the negative of the logarithm of the survival probability and is defined in (10) below. It is a component of the logistic transformation of the age-specific probability of death, and was called the half-logit in the working paper of Heathcote \& Puza (2007). Amongst its useful properties is the fact that for a cohort with Gompertz mortality, that is, a linear log hazard, the slope of the half logit is exactly the same as that of the log hazard (see Wang et al. 1998, p.134), but there is some change in the intercept. Thus, unlike certain other estimators of the hazard function based on grouped data, this quantity does not suffer from discretization bias, in the sense of perhaps falsely exhibiting deceleration. This is an important property of the half logit because, if it does indicate deceleration, then, for a homogeneous population, this could indeed reflect reality and not be an artifact of the method of estimation.

Müller et al. (1997) and Wang et al. (1998) are largely concerned with the limiting case in which the unit of discrete time becomes arbitrarily small. This is not of immediate relevance when using data published by official statistical agencies, and we take the unit of time as fixed. In addition, we ignore censoring. In Section 3, standard arguments are used to establish the asymptotic normality of the half logit estimator, and this has useful consequences for the estimation of the hazard function. The methods used are generally similar to those of Heathcote \& Puza (2005). 
The Australian Institute of Health and Welfare has recently published a comprehensive set of cause-of-death statistics for Australia during the twentieth century. Section 4 uses data stemming from this source to illustrate our methods by studying death from three causes in a population of elderly females. The final section treats a case of the deceleration of mortality and its subsequent re-acceleration. As mentioned above, given a population comprising a number of groups, each of which satisfies a Gompertz law of mortality, the question addressed is how different must the groups be in order that the population as a whole is not Gompertz in the sense of exhibiting deceleration of mortality? What emerges is that deceleration may be only temporary. We speculate that, if there are $k$ groups, then heterogeneity can be such that there are up to $k-1$ age intervals in which deceleration occurs, each followed by an interval of acceleration. We are able to resolve (numerically) only the case of three groups.

\section{Frailty models}

Frailty models were introduced by Vaupel et al. (1979) as a way of describing heterogeneity in a population. The frailty (susceptibility to death) of a randomly chosen individual is a non-negative random variable $Z$ with cumulative distribution function $F(z)$ and expectation $\mathrm{E}(Z)=1$. An expectation of unity defines what may be called a 'standard' individual. High realizations of $Z$ characterize frail members of the population, who tend to die at earlier ages, in contrast to more robust, less frail, individuals with low realizations of $Z$. Note that these models fix an individual's frailty at the initial age, a restriction that is well recognized.

If $x$ denotes age and $z$ frailty, then it is reasonable to suppose that the hazard function $\lambda(z, x)$ is non-decreasing in $z$ for each fixed $x$. A commonly made assumption is that of proportional hazards:

$$
\lambda(z, x)=z \lambda_{0}(x)
$$

with $\lambda_{0}(x)$ the baseline hazard. In particular, the Gamma-Gompertz model is extensively used (Vaupel et al., 1979; Horiuchi \& Coale, 1990; Steinsaltz \& Wachter, 2006, amongst others). Here $Z$ is gamma with the constraint of unit expectation and the baseline hazard is Gompertz, with $\lambda_{0}(x)=\exp (\alpha+b x)$.

A feature of this model is that it exhibits old-age deceleration in the population hazard function described by the logistic Perks formula, in which there is an approach to an asymptote as age increases (see appendix B of Horiuchi \& Coale, 1990). It should be mentioned at this stage that, in contrast to (1), a hazard function of the form

$$
\lambda(z, x)=\exp (\alpha+b z x)
$$

is used in Section 5.

The essentials of the frailty model are as follows. If $T(z)$ is the lifetime of an individual with frailty $z$ and hazard $\lambda(z, x)$, the probability of survival beyond age $x$ is

$$
\operatorname{Pr}(T(z)>x)=\exp (-\Lambda(z, x))
$$

where $\Lambda(z, x)=\int_{0}^{x} \lambda(z, u) d u$ is the cumulative hazard function for those of frailty $z$. Let $\lambda(x)$ denote the population hazard with associated cumulative hazard $\Lambda(x)=\int_{0}^{x} \lambda(u) d u$. Then a 
randomly chosen individual from the population has probability of survival beyond age $x$ of

$$
\exp (-\Lambda(x))=\int_{0}^{\infty} e^{-\Lambda(z, x)} d F(z)
$$

Differentiation yields the population hazard,

$$
\lambda(x)=-\frac{d}{d x} \log \left(\int_{0}^{\infty} e^{-\Lambda(z, x)} d F(z)\right)=\frac{\int_{0}^{\infty} \lambda(z, x) \exp (-\Lambda(z, x)) d F(z)}{\int_{0}^{\infty} \exp (-\Lambda(z, x)) d F(z)} .
$$

Allowing for notational changes, (4) is the same as (B5) of appendix B of Horiuchi \& Coale (1990), and, for proportional hazards, (3) is the same as (3) of Steinsaltz \& Wachter (2006).

Special cases of (3) will be of importance in this paper. The equation plays a central role in frailty models. Our description of heterogeneity is essentially the same as that of Vaupel et al. (1979). We later specialize to a mixture model that is (3) when $F(z)$ is a step function, $F(z)=\sum_{i \leq z} f_{i}$, thus dispensing with the normalization of mean frailty to unity. Then (3) can be written

$$
e^{-\Lambda(x)}=\sum_{i} f_{i} e^{-\Lambda_{i}(x)}
$$

This describes heterogeneity by a mixture of different groups. A generalization is to take frailty $f_{i}$ as $f_{i}(x)$, dependent on age $x$, an extension considered in Section 4.

Equation (5) is equally valid for unordered categorical groups that need not be interpretable as describing frailty. In this case the $f_{i}$ are initial prevalences of the different groups, a model discussed below in Section 5.

Equation (4) can also be expressed in terms of the density and distribution function of the lifetime random variable $T(z)$. If $G(z, x)$ is its distribution function and $g(z, x)$ the density then, using $g(z, x)=\lambda(z, x) \operatorname{Pr}(T(z)>x)$, (4) is the same as

$$
\lambda(x)=\frac{\int_{0}^{\infty} g(z, x) d F(z)}{\int_{0}^{\infty}(1-G(z, x)) d F(z)} .
$$

There may be circumstances when this formulation is useful in determining the asymptotic behaviour of $\lambda(x)$ at extreme old ages.

Returning to the proportional hazards model (1), observe that (4) can also be written as

$$
\lambda(x)=\frac{d}{d x} \log \left(\int_{0}^{\infty} e^{-z \Lambda_{0}(x)} d F(z)\right)=-\frac{d}{d x} \log F^{*}\left(\Lambda_{0}(x)\right),
$$

where $F^{*}(s)$ is the Laplace-Stieltjes transform of $F(z)$ and $\Lambda_{0}(x)$ is the baseline cumulative hazard. Carrying out the differentiation gives

$$
\lambda(x)=-\lambda_{0}(x) \frac{\left(F^{*}\left(\Lambda_{0}(x)\right)\right)^{\prime}}{F^{*}\left(\Lambda_{0}(x)\right)},
$$

an expression that is very convenient under models such as the Gamma-Gompertz. It is clear that a variety of forms for $\lambda(x)$ can be obtained by a suitable choice of baseline hazard and frailty distribution. 
These are the key formulae for models expressing the population hazard in terms of frailty. Equation (4) is the same as (B5) in Horiuchi \& Coale (1990), and (6) is the same as (4) of Steinsaltz \& Wachter (2006). However, (4) is valid for models other than proportional hazards. A case in point is the multiplicative Gompertz hazard (2).

A few general results can be deduced. For example, suppose that the minimum frailty occurs at a point $z_{m}$ of positive probability, $\operatorname{Pr}\left(Z=z_{m}\right)>0$, that $\lambda\left(z_{m}, x\right) \leq \lambda(z, x)$, and also that $\Lambda(z, x)-\Lambda\left(z_{m}, x\right)$ increases unboundedly with age $x$ uniformly in $z$. Then, from (4),

$$
\lambda(x)=\frac{\lambda\left(z_{m}, x\right) \operatorname{Pr}\left(Z=z_{m}\right) \exp \left(-\Lambda\left(z_{m}, x\right)\right)+\int_{z_{m}<z} \lambda(z, x) \exp (-\Lambda(z, x)) d F(z)}{\operatorname{Pr}\left(Z=z_{m}\right) \exp \left(-\Lambda\left(z_{m}, x\right)\right)+\int_{z_{m}<z} \exp (-\Lambda(z, x)) d F(z)} .
$$

Multiply the numerator and denominator by $\exp \left(\Lambda\left(z_{m}, x\right)\right)$ and take the limit as $x \rightarrow \infty$ to obtain the old-age approximation,

$$
\lambda(x) \sim \lambda\left(z_{m}, x\right)
$$

This is not a surprising result, as it states merely that, at very advanced ages, mortality in the population as a whole is dominated by that of the least frail, that is, by the strongest individuals. If $\lambda\left(z_{m}, x\right)$ is a constant independent of age (for example, if $z_{m}=0$ implies no ageing), then $\lambda(x)$ is asymptotically constant. This is consistent with the reliability approach of Gavrilov \& Gavrilova (2001) and partially with the Gamma-Gompertz model.

Note, however, that in the Gamma-Gompertz model as discussed by Horiuchi \& Coale (1990) with $\lambda(z, x)=A+z \exp (b x)$, the asymptote depends on $\lambda\left(z_{m}, x\right)=A, b$ and parameters of the gamma distribution. Hence (7) does not hold in this case where $z_{m}=0$ is approached continuously. Note also that the Gamma-Gompertz model, and more generally proportional hazards models, do not necessarily yield bounded $\lambda(x)$ if $z_{m}$ is strictly positive. Thus suppose that $0<z_{m} \leq z<\infty$. Then

$$
e^{-\Lambda(x)}=\int_{z_{m}}^{\infty} e^{-z \Lambda_{0}(x)} d F(z)=e^{-z_{m} \Lambda_{0}(x)} F_{1}^{*}\left(\Lambda_{0}(x)\right),
$$

where $F_{1}(y)$ is the distribution function of the translated frailty $Y=Z-z_{m}$ and $F_{1}^{*}(s)$ is its Laplace-Stieltjes transform. The hazard is now

$$
\lambda(x)=z_{m} \lambda_{0}(x)-\lambda_{0}(x) \frac{\left(F_{1}^{*}\left(\Lambda_{0}(x)\right)\right)^{\prime}}{F_{1}^{*}\left(\Lambda_{0}(x)\right)},
$$

which is consistent with (3.5) of Steinsaltz \& Wachter (2006). If the second term on the right is bounded, then again the old-age behaviour of the population hazard will be determined by $\lambda\left(z_{m}, x\right)=z_{m} \lambda_{0}(x)$. The important point here is that $z_{m}$ is strictly positive. It is reasonable to assume that this is the case with human populations because it is hard to envisage humans with zero frailty even if that value is assigned zero probability as in the Gamma-Gompertz model.

Intuitively, one can argue that, as weaker members of the population die off, it is possible that $\lambda(x)$ decelerates and then accelerates again according to the hazard of survivors, and that this process may be repeated. The example of Section 5 is a case in point. This supports our argument concerning old-age human mortality that Gamma-Gompertz and reliability theory models, although accurately describing deceleration, fail to account for the possibility of 
subsequent acceleration. The occurrence or not of this acceleration depends on the degree of heterogeneity in the population, as will be demonstrated in Section 5.

\section{The log transform}

Consider a cohort of $n$ lives, of whom $l(x)$ are expected to be alive at age $x$. We are interested in estimating quantities associated with this cohort using estimates based on aggregate data, as published by official statistical agencies, and available for ages $x=$ $0,1, \ldots, w$, where $w$ is the maximum age. The number $n=l(0)$ in the cohort is taken as known and large, whereas $l(x), 0<x<w+1, l(w+1)=0$, must be estimated from the aggregate data.

Let $\lambda(x)$ denote the population hazard function and

$$
p(x)=\exp \left(-\int_{0}^{x} \lambda(u) d u\right)
$$

the survivor function. The age-specific death rate at age $x$, that is, the probability of dying before age $x+1$ conditional on being alive at $x$, is

$$
q(x)=\frac{p(x)-p(x+1)}{p(x)}=1-\exp \left(-\int_{x}^{x+1} \lambda(u) d u\right),
$$

with $q(0)=1-p(1)$ and $q(w)=1$. In terms of expected frequencies this is

$$
q(x)=\frac{l(x)-l(x+1)}{l(x)} .
$$

For $x=0,1, \ldots, w$, the logarithmic transform of interest is

$$
\gamma(x)=-\log (1-q(x))=\int_{x}^{x+1} \lambda(u) d u,
$$

which can also be written

$$
\gamma(x)=\log \left(\frac{l(x)}{l(x+1)}\right)
$$

This is one half of the logistic transform of $q(x)$, and was called the half-logit in the working paper of Heathcote \& Puza (2007).

A study of old-age mortality using the logit of $q(x)$ can be found in Heathcote \& Higgins (2004). However, the half logit $\gamma(x)$ was demonstrated by Müller et al. (1997) and Wang et al. (1998) to possess superior descriptive properties at older ages. Like these authors, our main concern is with estimating $\lambda(x)$, but we differ in two respects: first regarding notation, in the sense that they write $q(x)$ for the death rate centred at the mid-point of $(x, x+1)$, and second and more importantly, our time interval is fixed, say at one year, whereas they were interested primarily in the case for which the interval becomes arbitrarily small.

Observe that for the Gompertz case, $\lambda(x)=\exp (\alpha+b x)$,

$$
\gamma(x)=\int_{x}^{x+1} e^{\alpha+b u} d u=\frac{e^{\alpha}}{b}\left(e^{b}-1\right) e^{b x},
$$


so that $\log (\gamma(x))$ has exactly the same slope as $\log (\lambda(x))$. Clearly, $b$ is the parameter of major interest in these models. Furthermore, the cumulative hazard $\Lambda(x)=\int_{0}^{x} \lambda(u) d u$ is

$$
\Lambda(x)=\int_{0}^{x} e^{\alpha+b u} d u=\frac{-e^{\alpha}}{b}+\frac{e^{\alpha+b x}}{b} .
$$

Thus estimates of $\log (\gamma(x))$ or $\Lambda(x)$ may be equally useful in studying the rate of old-age mortality in models based on Gompertz hazards, and both will be used below.

Let $\tilde{l}(x)$ be the random variable denoting the number of lives surviving at age $x$ of the cohort of $n$ individuals. A realization of this sequence of counts provides the data from which inferences are to be made concerning old-age mortality. It is convenient to assume the following:

The $n$ members of the cohort have independent and identically distributed lifetimes.

An immediate consequence is that $\tilde{l}(x), x=0,1, \ldots, w-1$, forms a sequence of binomial random variables with means

$$
\mathrm{E}(\tilde{l}(x))=n p(x)
$$

and second moments

$$
\operatorname{var}(\tilde{l}(x))=n p(x)(1-p(x)), \operatorname{cov}(\tilde{l}(x), \tilde{l}(y))=n(p(\max (x, y))-p(x) p(y)) .
$$

Here, $p(x)$ is the survivor function (8). Moreover, if $\tilde{l}$ is the $w \times 1$ random vector of the $\tilde{l}(x)$ and $\boldsymbol{l}$ its expectation determined by (13), then, as $n \rightarrow \infty, n^{-1 / 2}(\tilde{\boldsymbol{l}}-\boldsymbol{l})$ is asymptotically normally distributed with mean vector zero and covariance matrix $\mathbf{C}$ determined by (14).

These results will now be used to establish asymptotic normality of the sequence of estimators $\tilde{\gamma}(x)=\log (\tilde{l}(x) / \tilde{l}(x+1)), x=0,1,2, \ldots, w-1$. The argument follows that of Heathcote \& Higgins (2004) and Heathcote \& Puza (2005) for the logit of $q(x)$ and is a straightforward application of the Taylor expansion of $\log (z+1)=z-\left(z^{2} / 2\right)+\ldots$ for $|z|<$ 1 and $z=1$. It is the methodology applied to the estimation of Markov chains developed by Davis, Heathcote \& O'Neill (2002). We have

$$
\begin{aligned}
\tilde{\gamma}(x)-\gamma(x) & =\log \left(\frac{\tilde{l}(x)}{\tilde{l}(x+1)}\right)-\log \left(\frac{l(x)}{l(x+1)}\right) \\
& =\log \left(1+\frac{\tilde{l}(x)-l(x)}{l(x)}\right)-\log \left(1+\frac{\tilde{l}(x+1)-l(x+1)}{l(x+1)}\right) \\
& =\frac{\tilde{l}(x)-l(x)}{l(x)}-\frac{\tilde{l}(x+1)-l(x+1)}{l(x+1)}+o_{p}\left(n^{-1 / 2}\right) .
\end{aligned}
$$

Here $o_{p}\left(n^{-1 / 2}\right)$ is a random variable that, when divided by $n^{-1 / 2}$, tends to zero in probability as $n \rightarrow \infty$. In vector notation we have

$$
\tilde{\gamma}(x)-\gamma(x)=\left(0, \ldots, 0, \frac{1}{p(x)}, \frac{-1}{p(x+1)}, 0, \ldots, 0\right) \frac{1}{n}(\tilde{\boldsymbol{l}}-\boldsymbol{l})+o_{p}\left(n^{-1 / 2}\right) .
$$


Let $\tilde{\gamma}$ and $\boldsymbol{\gamma}$ be respectively the $w \times 1$ vectors of the $\tilde{\gamma}(x)$ and $\gamma(x)$ with $\tilde{\gamma}=\tilde{\gamma}(0)=$ $\log (\tilde{l}(0) / \tilde{l}(1))=\log (n / \tilde{l}(1))$. Furthermore, let $\mathbf{A}$ be the $w \times w$ matrix

$$
\mathbf{A}=\left(\begin{array}{cccccc}
-p(1)^{-1} & 0 & 0 & \cdots & 0 & 0 \\
p(1)^{-1} & -p(2)^{-1} & 0 & \cdots & 0 & 0 \\
0 & p(2)^{-1} & -p(3)^{-1} & & 0 & 0 \\
\vdots & & & \ddots & & \vdots \\
0 & 0 & 0 & & -p(w-1)^{-1} & 0 \\
0 & 0 & 0 & & p(w-1)^{-1} & -p(w)^{-1}
\end{array}\right) .
$$

Then $n^{1 / 2}(\tilde{\boldsymbol{\gamma}}-\boldsymbol{\gamma})=\mathbf{A} n^{-1 / 2}(\tilde{\boldsymbol{l}}-\boldsymbol{l})+o_{p}(1)$ and the asymptotic normality of $\tilde{\boldsymbol{l}}$ yields the following:

Result. As the cohort size $n$ tends to infinity, $n^{1 / 2}(\tilde{\boldsymbol{\gamma}}-\boldsymbol{\gamma})$ is asymptotically normally distributed with zero mean and covariance matrix $n^{-1} \mathbf{A C A}^{\mathrm{T}}=\operatorname{diag}\left(p(x+1)^{-1}-p(x)^{-1}, x=\right.$ $0,1,2, \ldots, w-1 ; p(0)=1)$, where $\mathbf{C}$ is the matrix given by (14).

That $n^{-1} \mathbf{A C A}^{\mathrm{T}}$ equals the asserted diagonal covariance matrix can be checked by carrying out the multiplication. The fact that the matrix is diagonal, and hence that the $\tilde{\gamma}(x)$ are asymptotically independent, is a useful consequence of the Markov property of the $\tilde{l}(x)$. A related result for the logit transformation was given by Davis et al. (2002).

Observe that, asymptotically,

$$
\begin{aligned}
\sigma^{2}(x) & =\operatorname{var}(\tilde{\gamma}(x))=\frac{1}{n}\left(\frac{1}{p(x+1)}-\frac{1}{p(x)}\right) \\
& =\frac{1}{l(x+1)}-\frac{1}{l(x)}=\frac{q(x)}{l(x+1)} \\
& =\frac{\text { probability of death in }(x, x+1) \text { given survival to } x}{\text { number of survivors to } x+1} .
\end{aligned}
$$

Hence, weights for a least squares fit to the half logits at age $x$ are

$$
\sigma^{-2}(x)=\frac{l(x+1)}{q(x)} .
$$

The question of estimation is taken up in the next section.

\section{An example}

The Australian Institute of Health and Welfare (AIHW) recently published in AIHW (2006) a study of major causes of death in Australia during the twentieth century. Generally five-year age intervals were used, but we obtained a selection of numbers of deaths owing to heart or circulatory disease, cancer and other causes by single years for selected cohorts. The data used in this and the next section are for 31147 women aged 74 in 1968 and 105 in 1999 and are classified by cause of death into the following three groups: 


\section{TABLE 1}

Numbers of survivors $l_{i}(x)$ at age $x$ for each $i=0,1,2,3$.

(For example, 3443 of the 31147 females aged 74 in 1968 would ultimately die of cancer. Of these 3443 , only five survived to 1995, and the last two died in 1998 aged 104)

\begin{tabular}{|c|c|c|c|c|c|c|}
\hline \multirow[b]{2}{*}{ Age } & \multirow[b]{2}{*}{$x$} & \multirow[b]{2}{*}{ Year } & \multicolumn{4}{|c|}{ Groups } \\
\hline & & & $\begin{array}{l}\text { All causes } \\
\quad l_{0}(x)\end{array}$ & $\begin{array}{c}\text { Heart disease } \\
\quad l_{1}(x)\end{array}$ & $\begin{array}{c}\text { Cancer } \\
l_{2}(x)\end{array}$ & $\begin{array}{c}\text { Other causes } \\
\quad l_{3}(x)\end{array}$ \\
\hline 74 & 0 & 1968 & 31147 & 21653 & 3443 & 6051 \\
\hline 75 & 1 & 1969 & 29738 & 20695 & 3244 & 5799 \\
\hline 76 & 2 & 1970 & 28266 & 19709 & 3007 & 5550 \\
\hline 77 & 3 & 1971 & 26648 & 18611 & 2787 & 5250 \\
\hline 78 & 4 & 1972 & 25192 & 17591 & 2579 & 5022 \\
\hline 79 & 5 & 1973 & 23635 & 16483 & 2392 & 4760 \\
\hline 80 & 6 & 1974 & 21978 & 15315 & 2181 & 4482 \\
\hline 81 & 7 & 1975 & 20293 & 14103 & 1991 & 4199 \\
\hline 82 & 8 & 1976 & 18819 & 13053 & 1795 & 3971 \\
\hline 83 & 9 & 1977 & 17219 & 11930 & 1591 & 3698 \\
\hline 84 & 10 & 1978 & 15593 & 10780 & 1402 & 3411 \\
\hline 85 & 11 & 1979 & 13999 & 9655 & 1219 & 3125 \\
\hline 86 & 12 & 1980 & 12520 & 8620 & 1031 & 2869 \\
\hline 87 & 13 & 1981 & 11038 & 7581 & 877 & 2580 \\
\hline 88 & 14 & 1982 & 9661 & 6587 & 741 & 2333 \\
\hline 89 & 15 & 1983 & 8340 & 5682 & 616 & 2042 \\
\hline 90 & 16 & 1984 & 7129 & 4821 & 507 & 1801 \\
\hline 91 & 17 & 1985 & 5988 & 4025 & 420 & 1543 \\
\hline 92 & 18 & 1986 & 4962 & 3303 & 343 & 1316 \\
\hline 93 & 19 & 1987 & 4004 & 2652 & 253 & 1099 \\
\hline 94 & 20 & 1988 & 3148 & 2065 & 176 & 907 \\
\hline 95 & 21 & 1989 & 2439 & 1592 & 124 & 723 \\
\hline 96 & 22 & 1990 & 1815 & 1174 & 89 & 552 \\
\hline 97 & 23 & 1991 & 1333 & 862 & 62 & 409 \\
\hline 98 & 24 & 1992 & 981 & 650 & 35 & 296 \\
\hline 99 & 25 & 1993 & 680 & 452 & 20 & 208 \\
\hline 100 & 26 & 1994 & 467 & 313 & 13 & 141 \\
\hline 101 & 27 & 1995 & 310 & 210 & 5 & 95 \\
\hline 102 & 28 & 1996 & 184 & 121 & 3 & 60 \\
\hline 103 & 29 & 1997 & 111 & 81 & 2 & 28 \\
\hline 104 & 30 & 1998 & 57 & 39 & 2 & 16 \\
\hline 105 & 31 & 1999 & 21 & 17 & 0 & 4 \\
\hline
\end{tabular}

- Group 1, being those alive in 1968 who ultimately die of heart or circulatory disease (ICD9 codes 390-459);

- Group 2, being those alive in 1968 who ultimately die of cancer (ICD9 codes 140-239); and

- Group 3, comprising the remainder of the 31147 women, dying of other causes.

For convenience, we call Group 0 (zero) those dying from all causes, that is, the full population of 31147 . With age 74 coded as age $x=0$, the $l_{i}(x)$ listed in Table 1 are the numbers alive in each of these groups $(i=0,1,2,3)$ at $x=0,1,2, \ldots, 31$. It is assumed that the 21 persons still alive at age 105 died before reaching their 106th birthday.

As in Müller et al. (1997) and Wang et al. (1998), local polynomial modelling (Fan \& Gijbels, 1996) was used to fit the half logit $\gamma(x)$, its derivative and logarithm for the three causes of death. After some experimentation, it was decided to use polynomials of order 2 
and the Epanechnikov kernel with bandwidth 5. Confidence intervals were obtained using the variance formula (3.6) in chapter 3 of Fan \& Gijbels (1996) and asymptotic normality. In each case, the weights used were taken as those in the matrix W of Fan \& Gijbels (1996, p. 58) multiplied by the weights at (15).

The fits are shown in Figure 1, and it is apparent that assuming the Gompertz model in all three cases is reasonable at least to age 100, although the fitted $\gamma(x)$ for Group 2 (cancer) in particular shows considerable instability at higher ages. We do not have an explanation for the apparent increased acceleration after age 100. What is clear is that there is no deceleration of mortality. This led us to postulate parametric Gompertz models of the form

$$
\log \gamma_{i}(x)=a_{i}+b_{i} x, i=1,2,3 .
$$

Table 2 shows the weighted least squares estimates of the $a_{i}$ and $b_{i}$.

Even though the three groups are not independent, in the absence of further information a plausible conclusion from Table 2 is that there is no significant difference between the slopes of the Gompertz fits for Groups 1, 2 and 3.

Figure 1 also shows plots for the full population in Group 0. Formula (18) of the next section was used to estimate $\gamma_{0}(x)$. Because $\gamma_{0}(x)=\log (l(x) / l(x+1))$, calculation using (18) or the methods just described lead to the same result, and the plots are included in Figure 1. We observe that a Gompertz model for Group 0 provides an acceptable fit, and least squares estimates of the parameters (standard errors in parentheses) give

$$
\hat{\alpha}_{0}=-3.467, \quad \hat{a}_{0}=-3.424(0.0190), \quad \hat{b}_{0}=0.085(0.0012) .
$$

The conclusion is that the Gompertz model provides an acceptable description of oldage mortality for the three causes of death separately, and for the population as a whole. There is no deceleration, and mortality continues to increase exponentially with age. In these circumstances of relative homogeneity, it seems that a mixture of Gompertz components leads to an overall Gompertz-type model. What will be shown below is that sufficient heterogeneity leads to deceleration and then exponential acceleration again.

\section{Heterogeneity and the deceleration of mortality}

As mentioned in Section 1, deceleration is an issue with a substantial literature, and here we touch on a small part of the field using variants of the data of the last section to explore the case in which there are three groups. As noted, the $b_{i}$ of Table 2 indicate that the cohort is fairly homogeneous in the sense that the slopes of the $\log \lambda_{i}(x), i \geq 1$, are not significantly different - hence the apparent absence of deceleration of mortality in the population as a whole. Heterogeneity of the $b_{i}, i \geq 1$, will induce deceleration, and the problem is to specify the degree of dispersion amongst the slope coefficients necessary for this to occur.

Note that the half logit $\gamma(x)$ for deaths from all causes can be written as a function of the half logits for specific causes. Suppose that there are $k$ exhaustive groups, with $l_{i}(x)$ the numbers of the $i$ th group alive at age $x$ and $\gamma_{i}(x)$ the corresponding half logit. Then (see (11)) a formula corresponding to (5) is

$$
\exp \left(-\gamma_{0}(x)\right)=\frac{l(x+1)}{l(x)}=\sum_{i=1}^{k} \frac{l_{i}(x)}{l(x)} \frac{l_{i}(x+1)}{l_{i}(x)}=\sum_{i=1}^{k} f_{i}(x) \exp \left(-\gamma_{i}(x)\right) .
$$



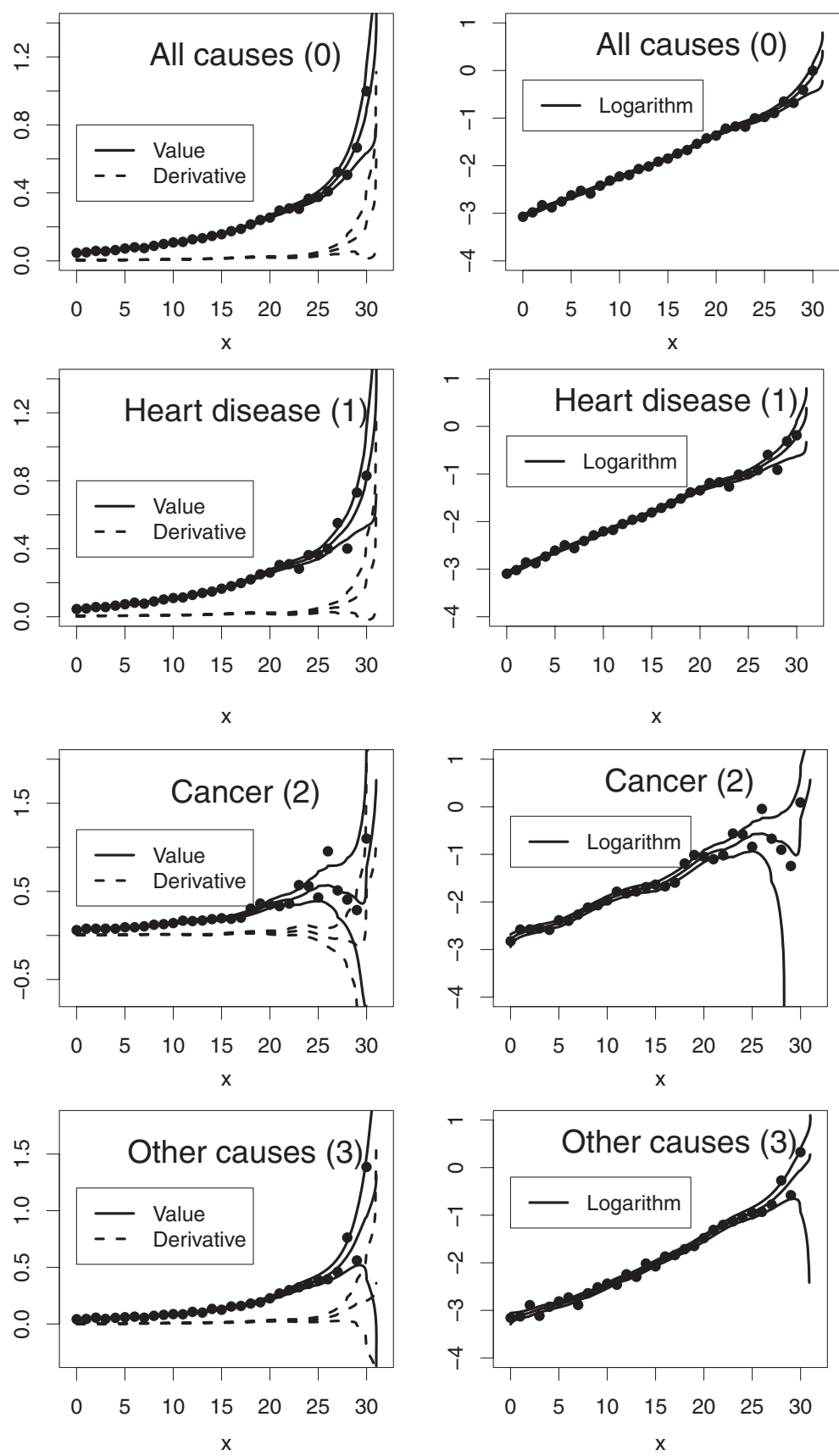

Figure 1. Estimated values, derivatives and logarithms of the half logit $\left(\gamma(x), \gamma^{\prime}(x)\right.$ and $\left.\log \gamma(x)\right)$ by all causes and by cause of death for Australian females born in 1894. Each triplet of lines shows point estimates and $95 \%$ confidence intervals for all ages from 74 to $105(x=0,1, \ldots, 31)$, and the dots show observed values. 
TABLE 2

Weighted least squares estimates of the $\alpha_{i}$ of (12) and the $a_{i}$ and $b_{i}$ of (16). Standard errors are in parentheses. Compare with (17) (results for Group 0)

\begin{tabular}{cccc}
\hline & 1 (Heart disease) & 2 (Cancer) & 3 (Other causes) \\
\hline$\hat{\alpha}_{i}$ & -3.473065 & -3.177743 & -3.700845 \\
$\hat{a}_{i}$ & -3.429584 & -3.135844 & -3.65568 \\
& $(0.019149)$ & $(0.039139)$ & $(0.04726)$ \\
$\hat{b}_{i}$ & 0.08634 & 0.08322 & 0.08966 \\
& $(0.00123)$ & $(0.00281)$ & $(0.00281)$ \\
\hline
\end{tabular}

Here, $f_{i}(x)=l_{i}(x) / l(x)$ is the proportion of individuals of group $i$ alive at age $x$. It may be possible to interpret the $f_{i}(x)$ as the frailty of group $i$ at age $x$. The problem can now be posed in the following way. Given that the $\gamma_{i}(x)$ on the right-hand side of (18) are all Gompertz and hence have the form $\gamma_{i}(x)=\exp \left(a_{i}+b_{i} x\right)$, with the $a_{i}$ and $b_{i}$ known, for which values of the $b_{i}$ does $\gamma_{0}(x)$ have a point of inflection? That is, which values of the $b_{i}$, $i \geq 1$, ensure that the equation $d^{2} \gamma(x) / d x^{2}=0$ has a solution in age $x$ ?

A complication is that the proportions $f_{i}(x)$ must also be differentiated. In fact, the data of Table 1 are sufficient to give numerical values for the $f_{i}(x)$, so that the right-hand side of (18) can be evaluated for each $x$. However, to place the discussion in the context of the standard frailty models of Section 2, in what follows we use the cumulative hazard $\Lambda(x)$ and (5).

Because the proportions $f_{i}=f_{i}(0)$ on the right-hand side of (5) do not depend on $x$, differentiation of

$$
\Lambda(x)=-\log \left(\sum_{i=1}^{k} f_{i} \exp \left(-\Lambda_{i}(x)\right)\right)
$$

is simpler than using $\gamma(x)$, and this is what is done below. For the $\Lambda_{i}(x)$ as on the right-hand side of (12) with the $f_{i}$ and the $\alpha_{i}$ and $b_{i}$ known, the problem now is to differentiate (19) twice, solve the equation

$$
\frac{d^{2}}{d x^{2}} \Lambda(x)=0,
$$

and find the set of $b_{i}$ for which a solution exists. It is this set of slope coefficients that specifies populations sufficiently heterogeneous for overall deceleration to occur, even though the hazard function of every individual is Gompertz.

We have not been able to solve this problem in any generality, but we do have a partial solution for the special case of $k=3$ groups. What emerges is the curious fact that (20) can have two or four solutions so that deceleration occurs and then at a later age a population Gompertz model reasserts itself. A consequence is that this is not consistent with the reliability approach expounded by Gavrilov \& Gavrilova (2001).

First consider the variation of one of the Gompertz slope coefficients $b_{i}$ about the average of the others, and find the set of these $b_{i}$ for which (20) has solutions. Using the $b_{i}, i=2,3$, of Table 2, choose $b_{1}$ according to the rule

$$
b_{1}=c \bar{b}_{-1}
$$




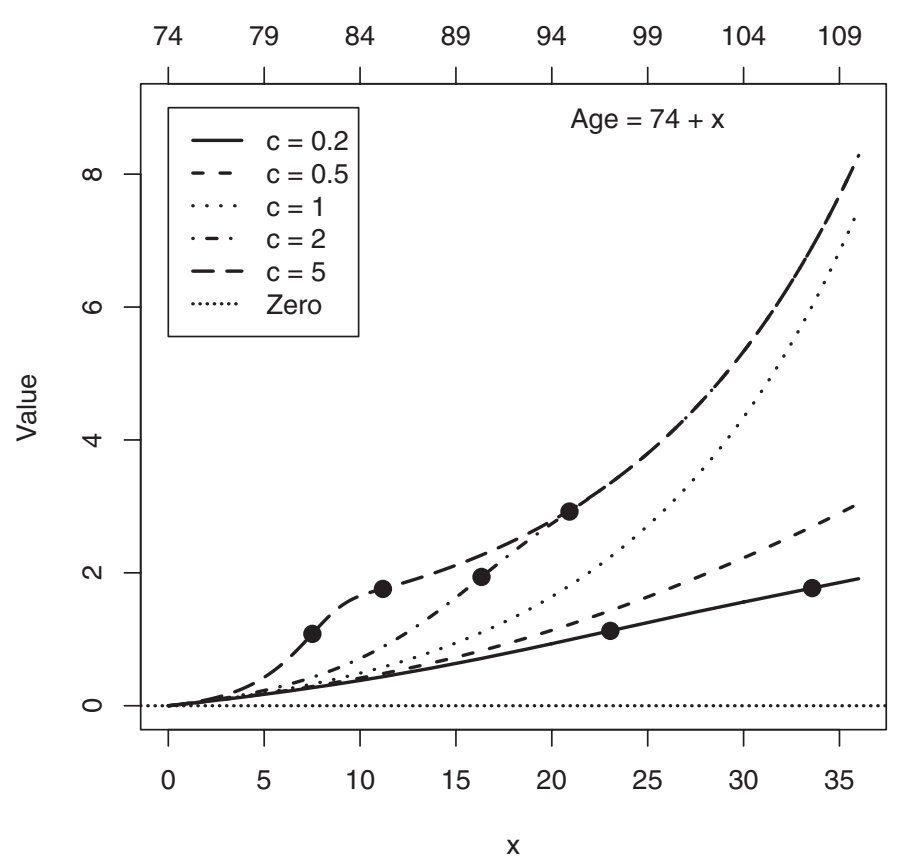

Figure 2. Values of the cumulative hazard function $\Lambda(x)$ of (19) with $\Lambda_{i}(x)$ as in (12) and Table 2 for selected values of $c$ as in (21). For each $c=0.2,2$ and 5, the dots show $\left(x_{0}, \Lambda\left(x_{0}\right)\right)$ and $\left(x_{1}, \Lambda\left(x_{1}\right)\right)$, where $x_{0}$ and $x_{1}$ are points of inflection of $\Lambda(x)$, that is, turning points of $\lambda(x)$.

where $\bar{b}_{-1}=\left(b_{2}+b_{3}\right) / 2=0.08644$ and $c$ is a multiple that can be varied. For a few selected values of $c$, Figure 2 shows the population cumulative hazard function $\Lambda(x)$ and marks the points $\left(x_{0}, \Lambda\left(x_{0}\right)\right)$ and $\left(x_{1}, \Lambda\left(x_{1}\right)\right)$ where $x_{0}$ and $x_{1}$ are solutions of (20) when such exist. Figure 3 shows the corresponding derivatives of the cumulative hazard function, namely $\lambda(x)=d \Lambda(x) / d x$, and the points $\left(x_{0}, \lambda\left(x_{0}\right)\right)$ and $\left(x_{1}, \lambda\left(x_{1}\right)\right)$.

Clearly, population deceleration and then acceleration set in only when $b_{1}$ deviates sufficiently in either direction from the mean of the other two groups. The interval $0.31<$ $c<1.59$ contains those values of $c$ for which $\Lambda(x)$ does not have a point of inflection, that is, for which there is insufficient heterogeneity to force deceleration. This interval features in Figure 4, which shows for values of $c$ from 0 to 5 the ages $x$ for which $d^{2} \Lambda(x) / d x^{2}$ is negative. For those $c$, heterogeneity is sufficient to force the population hazard function to decrease for ages in $\left(x_{0}, x_{1}\right)$. Table 3 gives numerical values of $x_{0}$ and $x_{1}$, and the cumulative hazard function and its derivative at these points, which are shown as dots in Figures 2 and 3.

The preceding discussion treats the case of one outlying group amongst the three. Now suppose that the dispersion of the $b_{i}$ is such that two of the three have values that may be considered far from a central value. In particular, keep $b_{2}$ fixed at 0.08322 and vary $b_{1}$ and $b_{3}$ according to

$$
b_{1}=r b_{2}, \quad b_{3}=b_{2} / r
$$

for different choices of $r$. As illustrated in Figure 5, there exist values of $r$ greater than 2 for which there are two intervals $\left(x_{0}, x_{1}\right),\left(x_{2}, x_{3}\right)$ in which the population hazard function 


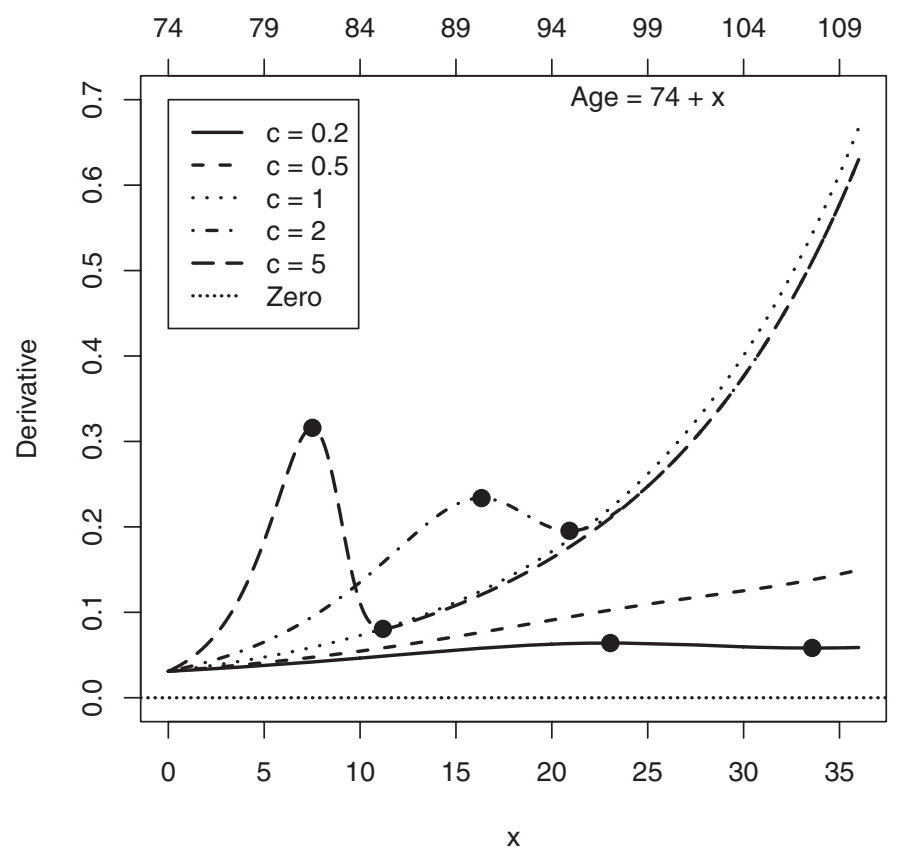

Figure 3. Derivatives of the cumulative hazard function $\Lambda^{\prime}(x)=\lambda(x)$, for selected values of $c$. For each $c=0.2,2$ and 5, the dots show $\left(x_{0}, \lambda\left(x_{0}\right)\right)$ and $\left(x_{1}, \lambda\left(x_{1}\right)\right)$, where $x_{0}$ and $x_{1}$ are points of inflection of $\Lambda(x)$, that is, turning points of $\lambda(x)$.

\section{TABLE 3}

Coordinates of the points marked by dots in Figures 2 and 3. The population hazard function $\lambda(x)=\Lambda^{\prime}(x)$ decreases for ages $x$ in the interval $\left(x_{0}, x_{1}\right)$. The degree of heterogeneity is determined by $c$ as in (21)

\begin{tabular}{lrrr}
\hline & \multicolumn{1}{c}{$c$} & \multicolumn{1}{c}{5.0} \\
\cline { 2 - 4 } & 0.2 & 2.0 & 7.51 \\
\hline$x_{0}$ & 23.05 & 16.33 & 11.19 \\
$x_{1}$ & 33.57 & 20.92 & 1.08 \\
$\Lambda\left(x_{0}\right)$ & 1.13 & 1.94 & 1.76 \\
$\Lambda\left(x_{1}\right)$ & 1.77 & 2.92 & 0.32 \\
$\Lambda^{\prime}\left(x_{0}\right)$ & 0.06 & 0.23 & 0.08 \\
$\Lambda^{\prime}\left(x_{1}\right)$ & 0.06 & 0.20 & \\
\hline
\end{tabular}

decreases. For moderate heterogeneity, for example $r=2$, there is only one such interval. Numerical values are given in Table 4. It can be shown that, for the three cases $1 \leq r<$ $1.21,1.21 \leq r<2.39$ and $r \geq 2.39$, there are zero, one and two intervals of deceleration, respectively.

An interpretation of these results is that the Gompertz group with $b$ a sufficiently large outlier in the (say) positive direction implies the existence of an age at which that group commences to die off rapidly, leading to a period of deceleration that ends when either the remaining groups reassert the overall Gompertz model or a new outlier appears and the process is repeated. If there are $k$ groups, then a plausible speculation is that, depending on the 


\section{TABLE 4}

Coordinates of the points marked by dots in Figure 5. The population hazard function decreases for ages $x$ in the intervals $\left(x_{0}, x_{1}\right)$ and $\left(x_{2}, x_{3}\right)$. The degree of heterogeneity is determined by $r$ as in (22)

\begin{tabular}{lccc}
\hline & & $r$ & \\
\cline { 2 - 4 } & 2 & 5 & 10 \\
\hline$x_{0}$ & 15.32 & 7.62 & 4.55 \\
$x_{1}$ & 24.12 & 11.95 & 6.94 \\
$x_{2}$ & - & 17.47 & 16.22 \\
$x_{3}$ & - & 31.17 & 33.36 \\
$\lambda\left(x_{0}\right)$ & 0.1825 & 0.2909 & 0.5008 \\
$\lambda\left(x_{1}\right)$ & 0.0824 & 0.0513 & 0.0415 \\
$\lambda\left(x_{2}\right)$ & - & 0.0554 & 0.0512 \\
$\lambda\left(x_{3}\right)$ & - & 0.0431 & 0.0331 \\
\hline
\end{tabular}

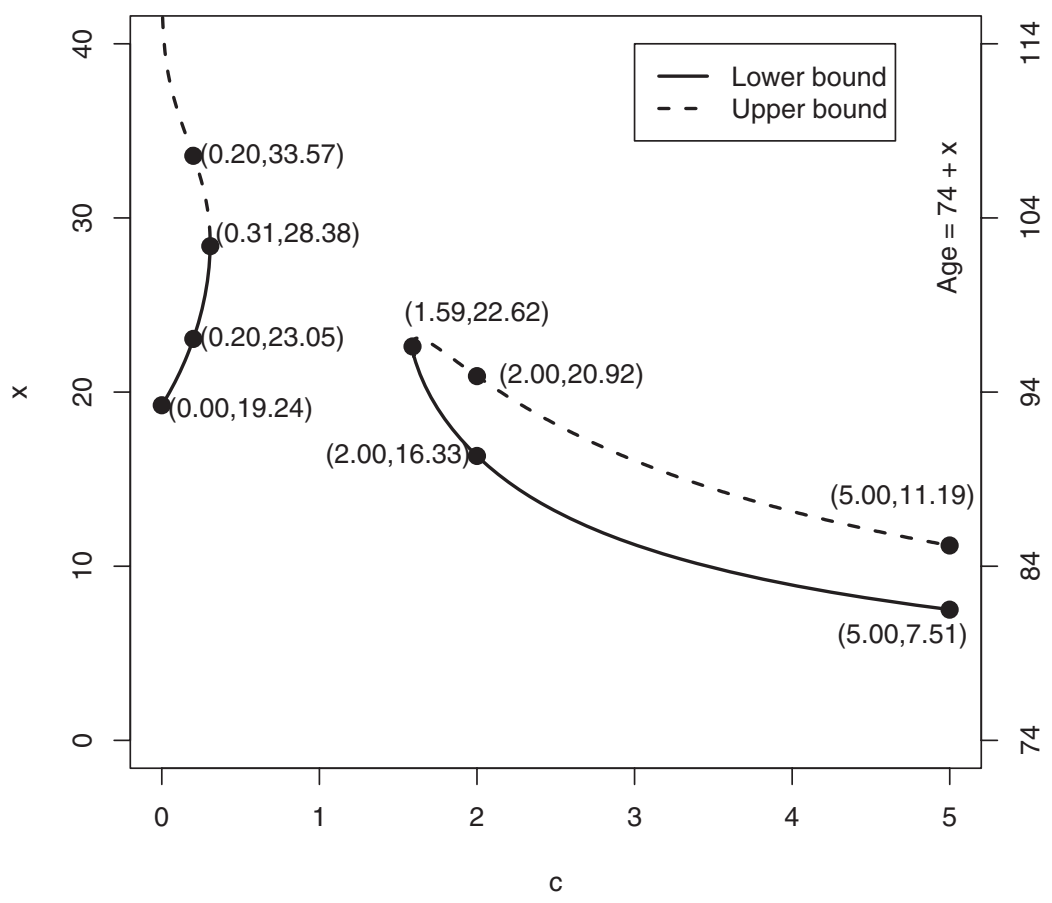

Figure 4. Lower and upper bounds of the ages for which the second derivative of the cumulative hazard function $\Lambda^{\prime \prime}(x)=\lambda^{\prime}(x)$ of (20) is negative, as a function of $c$, with points shown at selected values. $\lambda^{\prime}(x)$ is positive everywhere for $0.31<c<1.59$, and is negative for all $x>19.24$ if $c=0$.

nature of the heterogeneity, there are no or up to $k-1$ intervals in which decelaration occurs, followed by a return to a Gompertz-like increase in population mortality. This is consistent with the $k=2$ example on page 128 of Wang et al. (1998). The case of extreme heterogeneity leaves one group dominant at the oldest ages, and the dominance of that group forces ultimate Gompertz-like behaviour on the population. Of course, this may take place at an age too high 


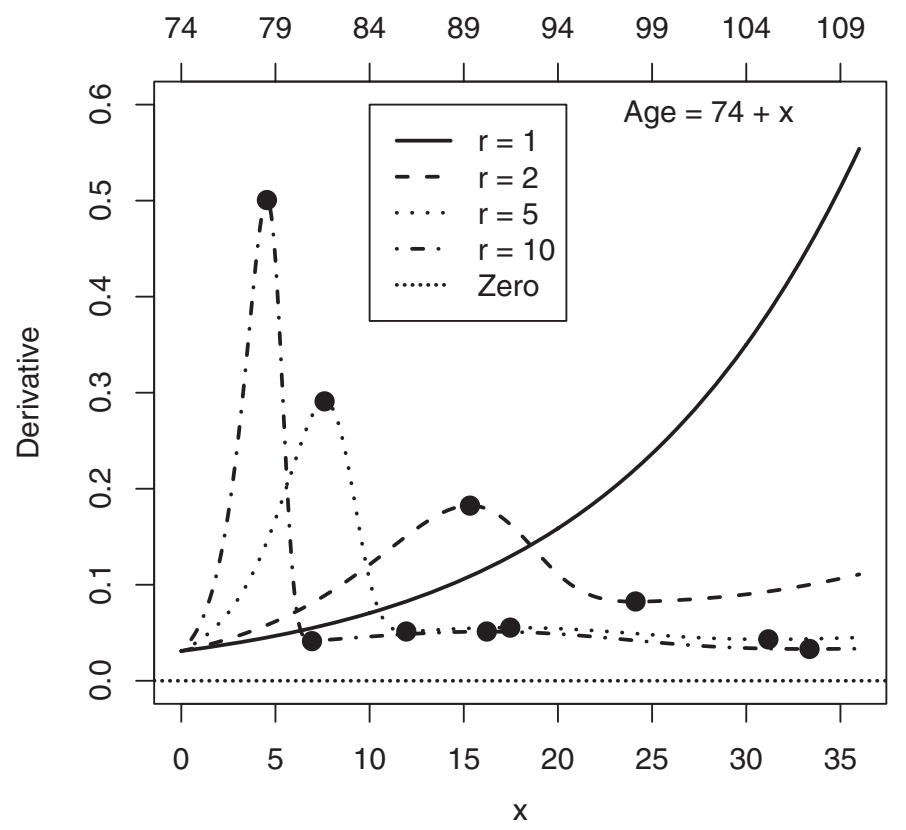

Figure 5. Derivatives of the cumulative hazard function $\Lambda^{\prime}(x)=\lambda(x)$, for selected values of $r$ as in (22). The dots show the points $\left(x_{i}, \lambda\left(x_{i}\right)\right)$, where the $x_{i}$ are points of inflection of $\Lambda(x)$.

to be of other than theoretical interest, but the important point is that our results suggest that deceleration can only be temporary for populations made up of Gompertz groups.

\section{References}

AIHW (2006). Mortality Over the Twentieth Century in Australia: Trends and Patterns in Major Causes of Death. AIHW cat. no. PHE 73. Canberra: Australian Institute of Health and Welfare.

CAREY, J.R. \& TUlJAPURKAR, S., eds (2003). Life span: evolutionary, ecological and demographic perspectives. Population and development review 29 (Supplement). New York: The Population Council.

Davis, B.A., HeATHCote, C.R. \& O'NeILl, T.J. (2002). Estimating and interpolating a Markov chain from aggregate data. Biometrika 89, 95-110.

FAN, J. \& GiJbels, I. (1996). Local Polynomial Modelling and its Applications. London: Chapman \& Hall.

GAVRILOV, L.A. \& GAVRILOVA, N.S. (2001). Regression modelling of aging and longevity. J. Theoret. Biol. 213, 527-545.

HEATHCOTE, C.R. \& Higgins, T. (2004). Regression modelling of mortality surfaces and the deceleration of mortality. Math. Popul. Stud. 11, 73-91.

HEATHCOTE, C.R. \& PUZA, B.D. (2005) Estimating processes of counts from cross-sectional aggregate data, with an application to multistate lifetables and health expectations. Aust. N. Z. J. Statist. 47, 425-439.

HeathCote, C.R. \& PuZA, B.D. (2007) The half logit and old age mortality. ANU College of Business and Economics, Working Paper 2007-01. http://www.ecocomm.anu.edu.au/research/ papers/papers.asp?disc=STAT.

HoRIUCHI, S. \& COALE, A.J. (1990). Age patterns of mortality for older women: an analysis using the age-specific rate of mortality change with age. Math. Popul. Stud. 2, 245-267.

HORIUCHI, S. \& WILMOTH, J.R. (1998). Deceleration in the age pattern of mortality at older ages. Demography 35, 391-412. 
MÜLLER, H.-G., WANG, J.-L. \& CAPRA, W.B. (1997). From life-tables to hazard rates: the transformation approach. Biometrika 84, 881-892.

STEINSALTZ, D.R. \& WACHTER, K.W. (2006). Understanding mortality rate deceleration and heterogeneity. Math. Popul. Stud. 13, 19-37.

VAuPEL, J.W., MANTON, K.G. \& Stallard, E. (1979). The impact of heterogeneity in individual frailty on the dynamics of mortality. Demography 16, 439-454.

WANG, J.-L., MÜLLER, H.-G. \& CAPRA, W.B. (1998). Analysis of oldest-old mortality: life tables revisited. Ann. Math. Statist. 26, 126-163. 